\title{
Quantification of skin lesions using high-frequency ultrasound and shear wave elastography in port-wine stain patients: a clinical study
}

\author{
Yuanjiao Tang ${ }^{1}$, Shan Cheng ${ }^{2}$, Xue Tang ${ }^{3}$, Ruiqian Guo ${ }^{1}$, Lingyan Zhang ${ }^{1}$, Li Qiu ${ }^{1}$ \\ ${ }^{1}$ Department of Ultrasound, West China Hospital of Sichuan University, Chengdu 610041, China; ${ }^{2}$ Department of Ultrasound, the Affiliated \\ Hospital of Guizhou Medical University, Guiyang 550004, China; ${ }^{3}$ Department of Thoracic Surgery, West China Hospital of Sichuan University, \\ Chengdu 610041, China \\ Contributions: (I) Conception and design: Y Tang, S Cheng, X Tang, L Qiu; (II) Administrative support: L Qiu; (III) Provision of study materials or \\ patients: X Tang, L Qiu; (IV) Collection and assembly of data: Y Tang, S Cheng, R Guo, L Zhang; (V) Data analysis and interpretation: L Qiu; (VI) \\ Manuscript writing: All authors; (VII) Final approval of manuscript: All authors. \\ Correspondence to: Li Qiu, MD. Department of Ultrasound, West China Hospital of Sichuan University, No. 37 Guo Xue Xiang, Chengdu 610041 , \\ China. Email: wsqiuli@126.com.
}

Background: This study aimed to assess the different types of port-wine stain (PWS) skin lesions quantitatively using high-frequency ultrasound (US) and shear wave elastography (SWE) before and after treatment, and investigate the feasibility and application value of high-frequency US and SWE in PWSs.

Methods: A total of 195 PWS patients with 238 skin lesions before treatment and 72 follow-up PWS patients with 90 skin lesions were assessed using high-frequency US and SWE. The skin lesions were divided into four groups: pink-type, purple-type, thickened-type, and nodular-type PWSs. Gray-scale US was used to observe normal skin, observe the skin changes of lesions, and assess the skin thickness. The thickened skin was calculated. Power Doppler (PD) signal grades were used to assess the skin blood signals. SW velocity (in $\mathrm{m} / \mathrm{s}$ ) and Young's elastic modulus (in $\mathrm{kPa}$ ) were used to assess the stiffness of normal skin and skin lesions. The heightened SWE was also calculated.

Results: The dermis hypoechogenicity, thickness of thickened skin, and skin PD signal grades were significantly higher in all PWS-type groups compared with the normal-skin group (all $\mathrm{P}<0.05$ ). The thickened skin and skin PD signal grades in the nodular-type and thickened-type group were significantly thicker and higher than those in the pink-type and purple-type group (all $\mathrm{P}<0.05$ ). The $\mathrm{PD}$ signal grades in the purple-type was significantly higher than that in the pink-type group $(\mathrm{P}<0.05)$. All SWE values of PWS lesions were significantly higher in the transverse section than those in the longitudinal section (all $\mathrm{P}<0.05$ ). The differences in heightened $\mathrm{E}_{\text {mean }}, \mathrm{E}_{\text {min }}$, $\mathrm{C}_{\text {mean }}$, and $\mathrm{C}_{\min }$ between each PWS group and the normal-skin group were not significant. The heightened $\mathrm{E}_{\max }$ and $\mathrm{C}_{\max }$ in the nodular-type PWS group was significantly higher than those in the normal-skin group and pinktype, and purple-type PWS groups (all $\mathrm{P}<0.05$ ). The heightened $\mathrm{E}_{\max }$ and $\mathrm{C}_{\max }$ were significantly higher in the thickened-type PWS group than those in the normal-skin group (all $\mathrm{P}<0.05$ ). In the evaluation of therapeutic effects, the ratio of dermis hypoechogenicity in pink-type lesions significantly decreased, and thickened skins in all groups were significantly thinned (all $\mathrm{P}<0.05$ ). The differences of $\mathrm{PD}$ signal grades, heightened $\mathrm{E}_{\max }$, and $\mathrm{C}_{\max }$ in all groups between pre-treatment and post-treatment showed no significance.

Conclusions: High-frequency US and SWE show feasibility and application values assessing PWS skin lesions. Their features include dermis hypoechogenicity, thicker skin, higher PD signal grades, higher $\mathrm{E}_{\max }$, and higher $\mathrm{C}_{\max }$. Thicker skin is thus the best feature for assessing therapeutic effect.

Keywords: High-frequency ultrasound (high-frequency US); port-wine stain (PWS); shear wave elastography (SWE); treatment

Submitted Sep 26, 2019. Accepted for publication Nov 28, 2019.

doi: $10.21037 /$ atm.2019.12.57

View this article at: http://dx.doi.org/10.21037/atm.2019.12.57 


\section{Introduction}

Port-wine stains (PWSs) are progressive vascular malformations characterized by ectatic capillary- and venule-sized vessels in the papillary and upper reticular dermis. They occur in $0.3-0.5 \%$ of the population and are predominantly localized in the head-and-neck region and sometimes in the trunk and limbs $(1,2)$. The skin color of patients with a PWS can range from light pink to dark purple, and the lesions can be divided into a pinktype, purple-type, thickened-type, and nodular-type PWS based on clinical manifestations. PWSs are associated with both physical and psychological problems because they can result in disfigurement, while the development of nodules increases the risk of spontaneous bleeding (3). Therefore, timely treatment is essential to decrease cosmetic and psychological sequelae. Clinically, different type lesions present different therapeutic efficacies, and precise therapeutic effect assessment could help to choose the appropriate treatment and avoid repeated treatment. The most common method to assess lesions' therapeutic efficacies is to take pictures of the lesions by standardizing the shooting conditions to form a standardized visual score. Though standardized visual score has the advantages of cheaper cost, simplicity, and intuitiveness, it can be influenced by the subjective nature of the evaluator and the shooting technique, and needs to be supplemented with more objective evaluation methods.

Despite being the gold standard for PWS diagnosis and assessment, excisional biopsy has the disadvantage of being highly invasive which can lead to secondary effects complicating the lesion monitoring and treatment. Therefore, a noninvasive diagnostic method is preferred. Magnetic resonance imaging (MRI) and computed tomography (CT) findings of PWSs are subtle, with skin thickening and occasional increased subcutaneous thickness as the only findings (4). Their costs are also too high for routine use. Optical coherence tomography is an optical technique that enables high-resolution in vivo imaging of subsurface structures. However, its maximum detection depth in the skin is approximately $1 \mathrm{~mm}$, which prevents the detection of structures and blood vessels in the deeper dermis (5).

High-frequency ultrasound (US) is a noninvasive method proven to be helpful in diagnosing and assessing superficial lesions $(6,7)$. High-frequency US is advantageous for the study of the skin because it allows the detailed visualization of dermal and subcutaneous anatomy and can detect power
Doppler (PD) blood signals in lesions. Furthermore, its advantages including high resolution, reproducibility, low cost, and real-time results make it more practical compared with other methods in assessing skin lesions. In a recent study, Gong et al. applied high-frequency US to evaluate PWS lesions, and found that US could evaluate the depth and hemodynamics, which may help clinicians optimize laser intensity parameters for treatment of an individual patient and predict the effect (6). However, they did not assess PWS lesions before and after treatment. US elastography is a noninvasive method that creates an image of the mechanical characteristics of the tissue including elasticity and stiffness. It has been applied to assess the stiffness of skin lesions and has proven to be successful for detecting and assessing skin elastic changes related to diseases or physiologic processes $(8,9)$. Shear wave elastography (SWE) is a new US elastography technique, and compared with the traditional strain elastography, SWE has the advantages of being reproducible, quantitative, operator-independent, and capable of producing real-time results. In previous studies, SWE has been used to assess sclerosis skin lesions; it was demonstrated to be more sensitive in detecting subtle skin changes compared with gray-scale US and could reflect the degree of skin involvement (10-12). On our previous studies $(13,14)$, the elasticity of healthy skin was quantitatively assessed using SWE, demonstrating good reliability and feasibility of SWE in measuring skin elasticity. However, no previous study has examined the quantification of skin lesions using SWE in PWS patients.

The purpose of this study was to assess the different types of PWS skin lesions quantitatively using high-frequency US and SWE before and after treatment, and to investigate the feasibility and application values of high-frequency US and SWE in PWSs.

\section{Methods}

\section{Patient population}

This study comprised 195 PWS patients with a total of 238 skin lesions before treatment. The patients were recruited from the Department of Dermatology in West China Hospital, Sichuan University, from March 2017 to March 2019. The study was approved by the ethics committee of West China Hospital, Sichuan University. All participants were provided with informed consent forms before they underwent US and SWE examinations to explain that their imaging data would be used for scientific 

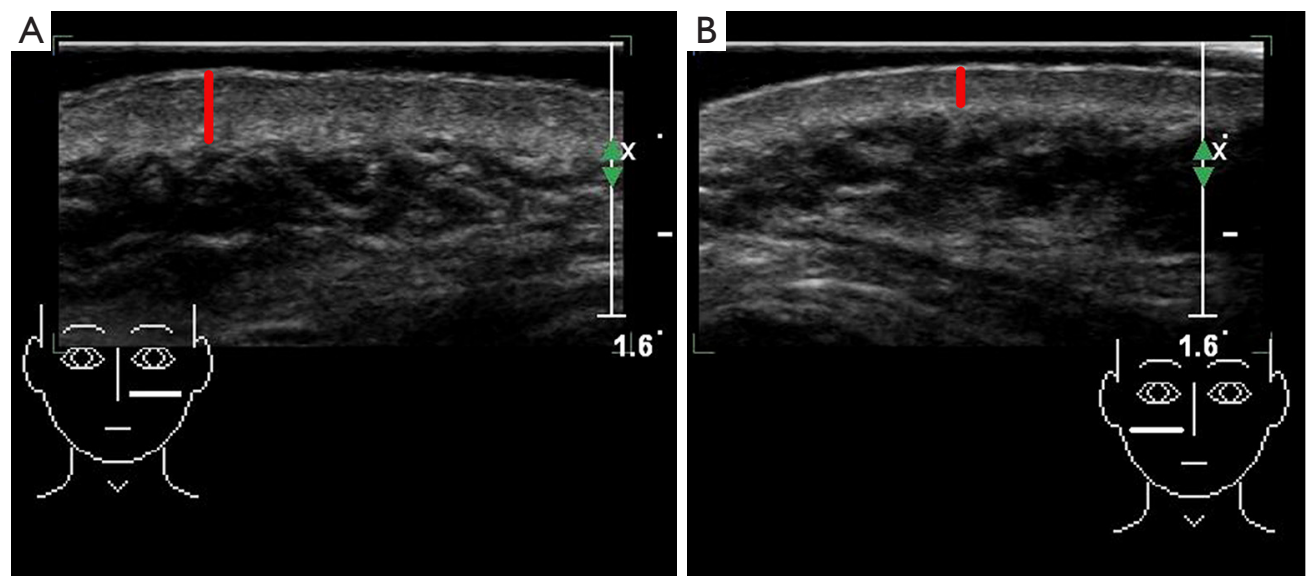

Figure 1 Gray-scale US used to assess the skin thickness (red line) of PWS lesions on the left face (A) and the normal side on the right face (B). PWS, port-wine stain; US, ultrasound.

research. The inclusion criteria were as follows: (I) patients diagnosed with PWSs pathologically based on skin biopsy by a dermatologist; (II) patients older than 1 year; and (III) patients with good compliance. The exclusion criteria were as follows: (I) pregnancy; (II) a current or previous history of any systemic disease or other dermal diseases; (III) scar, inflammation, or tumor in skin lesions; and (IV) bad compliance. The skin lesions were divided into four groups by sophisticated dermatologists: pink-type, purple-type, thickened-type, and nodular-type PWS groups. After onetime photodynamic therapy, the follow-up PWS patients were enrolled.

\section{High-frequency US examination}

US examinations were performed with an ultrasonic device (SuperSonic Imagine, Aix-en-Provence, France). The probe frequency was $12-15 \mathrm{MHz}$, and the superficial condition was selected. The probe surface was covered with several millimeters of US gel and placed perpendicularly to the skin to enable total contact causing no pressure on the skin. Gray-scale US was used to observe skin changes and assess the skin thickness of each lesion and the normal side. The largest depth of the skin was recorded (Figure 1), and the thickness of the thickened skin (lesion-skin thickness - normal-skin thickness) was calculated. Afterward, PD US was used to assess the blood signals of skin lesions and the normal sides. The color gain was increased until a background noise appeared and then was reduced until the noise was suppressed, thus ensuring maximum sensitivity. PD signals of skin lesions were scored as follows: grade
$0=$ absence, no color signals; grade $1=$ mild, one or two punctate color signals; grade $2=$ moderate, three or four punctate color signals or one stripe color signal; and grade $3=$ marked, more than five punctate color signals or more than two stripe color signals (Figure 2) (15). US scanning was performed by two adept radiologists with 7 and 10 years of experience in superficial tissue US imaging. The imaging experts were blinded to patient data. Before the study, the investigators reached a consensus on the adoption of US scanning technique and data interpretation. A diagnostic consistency test was conducted, and a $\kappa$ value of 0.9 was obtained. Briefly, the skin lesions of 10 PWS patients were evaluated by the 2 radiologists, and the ultrasonic features were observed.

\section{SWE examination}

SWE (SuperSonic Imagine, Aix-en-Provence, France) was used to assess the skin stiffness of each lesion and the normal side. The probe frequency was $12-15 \mathrm{MHz}$, and the superficial condition was selected. The probe surface was covered with several millimeters of US gel and placed perpendicularly to the skin in both transverse and longitudinal sections. SWE images were displayed together with the gray-scale US images. A square region of interest (ROI) was preset for obtaining the SWE images, and the ROIs included the skin and the subcutaneous layers. In the ROI, tissue stiffness was delineated using color mapping in which very soft tissues were coded in dark blue, with areas of increasing stiffness coded in light blue, green, orange, and red. At each site, the transducer was held for around 

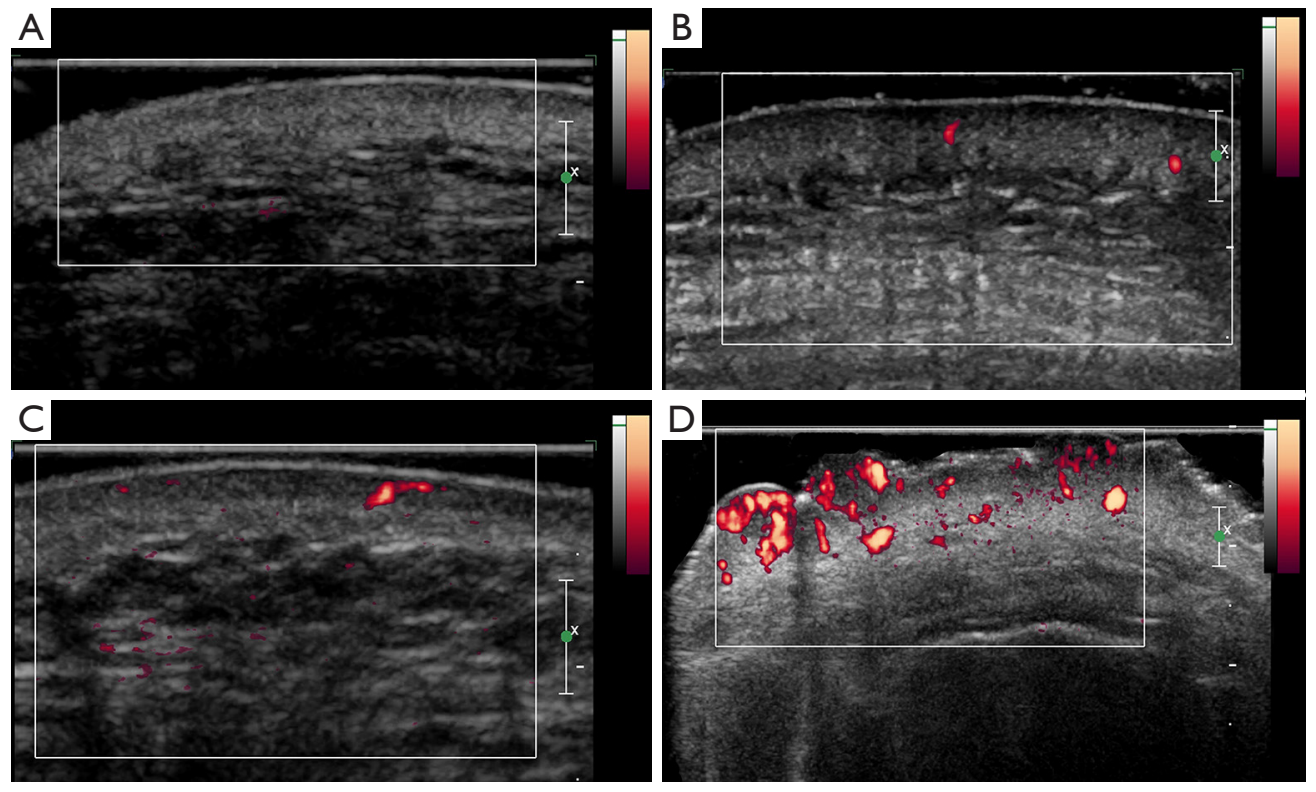

Figure 2 Blood signals of PWS lesions assessed using PD ultrasound. (A) Grade 0 of a pink-type lesion; (B) grade 1 of a thickened-type lesion; (C) grade 2 of a thickened-type lesion; (D) grade 3 of a nodular-type lesion. PD, power Doppler.
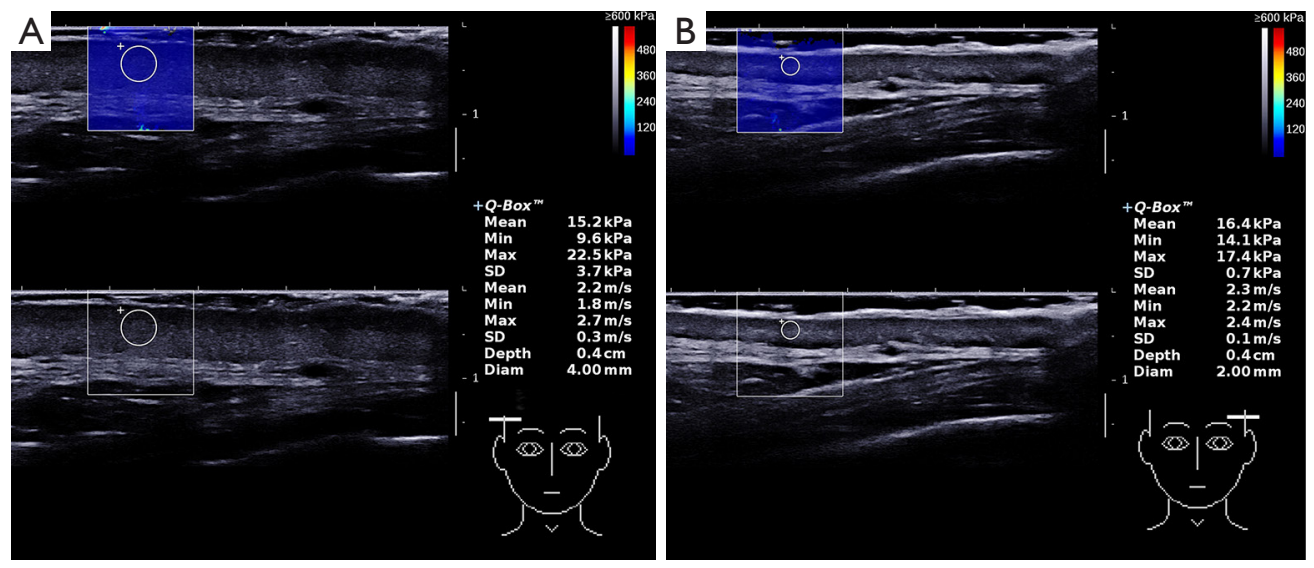

Figure 3 SWE used to assess the skin stiffness of PWS lesions on the right temple (A) and the normal side on the left temple (B). PWS, port-wine stain; SWE, shear wave elastography.

$10 \mathrm{~s}$ until a stable color was obtained in the ROI area. A round Q-box (a small circle of diameter 1-4 mm) was adjusted to the skin thickness. SW velocity (in $\mathrm{m} / \mathrm{s}$ ) and Young's elastic modulus (in $\mathrm{kPa}$ ) were automatically calculated using the US system (Figure 3). The heightened SWE value (lesion-skin SWE value - normal-skin SWE value) was calculated. SWE scanning was performed by two adept radiologists with 2 years of experience in SWE imaging who were not the two US scanners. The imaging experts were blinded to the patient data. Before the study, the investigators reached a consensus on the adoption of the SWE scanning technique and data interpretation. A diagnostic consistency test was conducted, and a $\kappa$ value of 0.85 was obtained. Briefly, the skin lesions of 10 PWS patients were evaluated by the 2 radiologists, and the skin stiffness values were obtained.

All measurements were carried out three times, and the averages of the thickness and SWE values and the highest 
Table 1 Patient characteristics

\begin{tabular}{lcccc}
\hline Variables & Pink type $(n=118)$ & Purple type $(n=50)$ & Thickened type $(n=58)$ & Nodular type $(n=12)$ \\
\hline Age, year, range & $10-65$ & $18-75$ & $20-80$ & $49-89$ \\
Age, year & $35.56 \pm 14.86$ & $52.44 \pm 11.91^{*}$ & $56.30 \pm 11.46^{\star}$ & $68.58 \pm 11.90^{\star, * \$ \$}$ \\
Male, \% & $54(49.15 \%)$ & $25(50.00 \%)$ & $31(53.45 \%)$ & $7(58.33 \%)$ \\
BMI, $\mathrm{kg} / \mathrm{m}^{2}$ & $19.45 \pm 14.29$ & $20.58 \pm 3.16$ & $21.07 \pm 2.77$ & $22.23 \pm 2.60$ \\
\hline
\end{tabular}

${ }^{*}, \mathrm{P}<0.01$ versus the pink-type group; ${ }^{\#}, \mathrm{P}<0.01$ versus the purple-type group; ${ }^{\$}, \mathrm{P}<0.01$ versus the thickened-type group. $\mathrm{BMI}$, body mass index; PWS, port-wine stain.

Table 2 Comparisons of ultrasonic features among groups

\begin{tabular}{|c|c|c|c|c|c|}
\hline Ultrasonic features & Normal skin $(n=238)$ & Pink type $(n=118)$ & Purple type $(n=50)$ & Thickened type $(n=58)$ & Nodular type $(n=12)$ \\
\hline Skin nodularity & $0(0.00 \%)$ & $0(0.00 \%)$ & $0(0.00 \%)$ & $0(0.00 \%)$ & $12(100.00 \%)^{\alpha_{1, x, \#, \$}}$ \\
\hline Thickened skin (mm) & $0.00 \pm 0.00$ & $0.35 \pm 0.36^{\&}$ & $0.56 \pm 0.44^{\&}$ & $0.94 \pm 0.59^{\varepsilon_{1, x, \#}}$ & $3.98 \pm 2.20^{\ell_{1, * *}, \$ \$}$ \\
\hline \multicolumn{6}{|l|}{ PD signal grades } \\
\hline 1 & 0 & 4 & 4 & 5 & 1 \\
\hline 2 & 0 & 5 & 9 & 18 & 5 \\
\hline 3 & 0 & 5 & 4 & 11 & 3 \\
\hline
\end{tabular}

${ }^{\&}, \mathrm{P}<0.01$ versus the normal-skin group; ${ }^{*}, \mathrm{P}<0.01$ versus the pink-type group; ${ }^{*}, \mathrm{P}<0.01$ versus the purple-type group; ${ }^{\$}, \mathrm{P}<0.01$ versus the thickened-type group.

PD signal grades were used for statistical analysis.

\section{Statistical analysis}

The SPSS 22.0 (SPSS Inc.) software was used for data analysis, with $\mathrm{P}<0.05$ considered statistically significant. The measurement data were compared using one-way analysis of variance (ANOVA), independent-sample $t$-test, or paired-sample $t$-test. Enumeration data were compared using the Pearson's chi-square test or McNemar's test. The ranked data were assessed using the Mann-Whitney $\mathrm{U}$ test or Wilcoxon test.

\section{Results}

\section{Patient characteristics}

This study included 195 congenital PWS patients comprising a total of 238 skin lesions before treatment. Of these, 156 lesions were located in the facial region, 31 in the head-and-neck regions, and 51 in the extremity and trunk regions. The main characteristics of the study patients are shown in Table 1. The nodular-type patients were older than the three other patient types. The purpletype and thickened-type patients were significantly older than the pink-type patients (both $\mathrm{P}<0.01$ ). The gender ratios and the body mass indexes (BMIs) showed no significant differences among the groups (all $\mathrm{P}>0.05$ ). Of all the patients, 64 cases chose to abandon treatment, 72 cases accepted photodynamic therapy, 30 cases accepted other therapies, and 29 cases were lost to follow-up. After one-time photodynamic therapy, the 72 follow-up PWS patients with a total of 90 skin lesions were enrolled. Of these, 72 lesions were located in the facial region, 9 in the head-and-neck regions, and 9 in the extremity and trunk regions.

\section{High-frequency US examination}

The ultrasonic features among the groups are shown in Table 2.

Compared with the normal-skin group, all PWS-type 
groups showed dermis hypoechogenicity, with significant differences (all $\mathrm{P}<0.05$ ). This ultrasonic feature was detected in $100 \%$ patients with purple-type, thickened-type, and nodular-type PWSs, and $83.89 \%$ of patients with pink-type PWSs.

Skin nodularity was detected in only nodular-type PWS skin lesions among the groups, with significant differences (all $\mathrm{P}<0.05$ ). Moreover, the hypoechoic nodule could be detected in every nodular-type lesion.

Compared with the normal skin, the skin of all PWStype lesions were thickened, with significant differences (all $\mathrm{P}<0.05$ ). The thickened skin in the nodular-type group measured $3.98 \pm 2.20 \mathrm{~mm}$, which was the thickest among all the groups and significantly thicker than that in the other three PWS groups (all $\mathrm{P}<0.05)$. The thickened skin in the thickened-type group measured $0.94 \pm 0.59 \mathrm{~mm}$, which was significantly thicker than that in the pink-type and purpletype groups (both $\mathrm{P}<0.05$ ). The thickened skin in the pinktype and purple-type groups measured $0.35 \pm 0.36 \mathrm{~mm}$ and $0.56 \pm 0.44 \mathrm{~mm}$, respectively. Although the thickened skin in the purple-type group was thicker than that in the pinktype group, the difference between these two groups was not significant $(\mathrm{P}>0.05)$.

In the normal skins, no PD signal was detected. The skin PD signal grades in all PWS-type groups were higher than those in the normal-skin group, with significant differences (all $\mathrm{P}<0.01$ ). The $\mathrm{PD}$ signal grades in the purple-type, thickened-type, and nodular-type groups were all significantly higher than those in the pink-type group (all $\mathrm{P}<0.01$ ). Meanwhile, significantly higher skin $\mathrm{PD}$ signal grades were found in the thickened-type and nodulartype groups compared with the pink-type and purple-type groups (all $\mathrm{P}<0.01)$. However, the difference in skin PD signal grades between the thickened-type and nodular-type groups was not significant $(\mathrm{P}>0.05)$.

\section{SWE examination}

Comparisons of SWE values in transverse and longitudinal sections are shown in Figure 4. All SWE values $\left(\mathrm{E}_{\text {mean }}, \mathrm{E}_{\text {min }}\right.$, $\mathrm{E}_{\max }, \mathrm{C}_{\text {mean }}, \mathrm{C}_{\min }$, and $\mathrm{C}_{\max }$ ) of PWS lesions were higher in the transverse section than those in the longitudinal section, with significant differences (all $\mathrm{P}<0.05$ ).

As all SWE values of PWS lesions were higher in the transverse section than those in the longitudinal section. We compared the heightened SWE values in the transverse section among the groups, and the results are shown in Table 3. Although all heightened SWE values in all PWS- type groups were higher than those in the normal-skin group, the differences in heightened $\mathrm{E}_{\text {mean }}, \mathrm{E}_{\text {min }}, \mathrm{C}_{\text {mean }}$, and $\mathrm{C}_{\text {min }}$ between each PWS group and the normal-skin group were not significant. Meanwhile, the heightened $\mathrm{E}_{\text {mean }}$, $\mathrm{E}_{\text {min }}, \mathrm{C}_{\text {mean }}$, and $\mathrm{C}_{\min }$ among all PWS groups showed no significant differences neither. Nevertheless, the heightened $\mathrm{E}_{\max }$ and $\mathrm{C}_{\max }$ in the nodular-type PWS group were the highest among all groups and significantly higher than those in the normal-skin group and pink-type and purpletype PWS groups (all $\mathrm{P}<0.05$ ). The heightened $\mathrm{E}_{\max }$ and $\mathrm{C}_{\max }$ were higher in the thickened-type PWS group than those in the normal-skin group, with significant differences (both $\mathrm{P}<0.05$ ).

\section{Comparisons of ultrasonic features and SWE values between pre-treatment and post-treatment}

According to the above findings, we compared all ultrasonic features and heightened $\mathrm{E}_{\max }$ and $\mathrm{C}_{\max }$ in transverse sections between pre-treatment and post-treatment of all PWS groups after one-time photodynamic therapy with the results summarized in Table 4. In the pink type group, $73.17 \%$ of lesions showed dermis hypoechogenicity before treatment, and the ratio decreased to $46.34 \%$ after treatment, with the difference being significant $(\mathrm{P}<0.05)$. However, the differences of dermis hypoechogenicity in the other three groups between pre-treatment and posttreatment showed no significance. For skin nodularity in the nodular type group, the difference between pretreatment and post-treatment also had no significance. However, thickened skins in all groups were significantly thinned between pre-treatment and post-treatment (all $\mathrm{P}<0.05)$. After treatment, thickened skin ranged from $0.37 \pm 0.27$ to $0.23 \pm 0.19 \mathrm{~mm}$ in the pink type group, from $0.71 \pm 0.51$ to $0.47 \pm 0.41 \mathrm{~mm}$ in the purple type group, from $0.94 \pm 0.72$ to $0.71 \pm 0.58 \mathrm{~mm}$ in the thickened type group, and from $3.69 \pm 3.77$ to $1.60 \pm 0.92 \mathrm{~mm}$ in the nodular type group. Nevertheless, the differences of PD signal grades, heightened $\mathrm{E}_{\max }$, and $\mathrm{C}_{\max }$ in all groups between pretreatment and post-treatment showed no significance.

\section{Discussion}

In this study, high-frequency US and SWE were used to quantitatively assess different types of PWS skin lesions before and after treatment, and the feasibility and application values of high-frequency US and SWE in PWSs were investigated. 

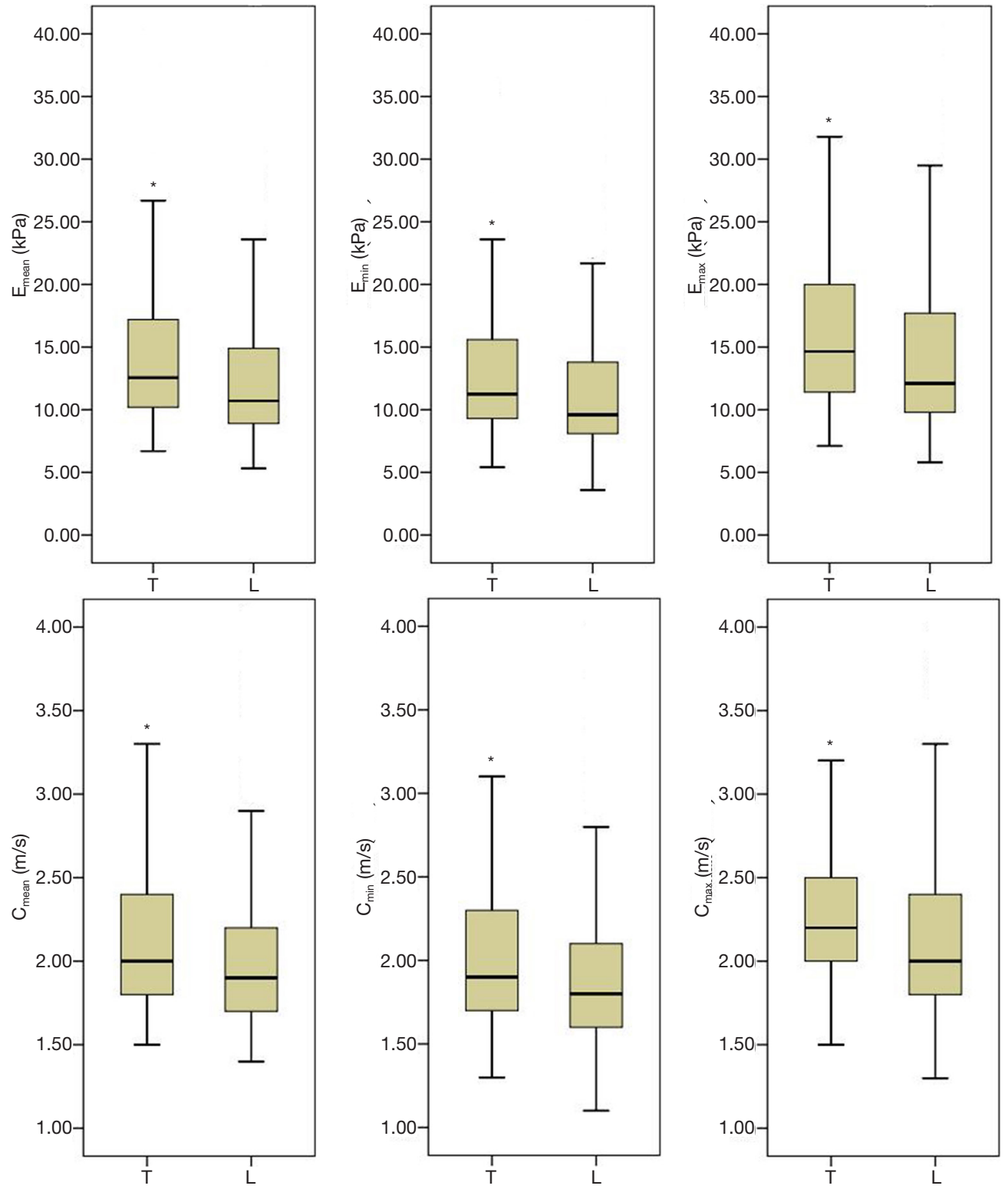

Figure 4 Box-and-whisker plots of $\mathrm{E}_{\text {mean }}, \mathrm{E}_{\min }, \mathrm{E}_{\max }, \mathrm{C}_{\text {mean }}, \mathrm{C}_{\min }$, and $\mathrm{C}_{\max }$ of $\mathrm{PWS}$ lesions in transverse and longitudinal sections. The top and bottom of each box represent the $75^{\text {th }}$ percentile and $25^{\text {th }}$ percentile, respectively; the horizontal line in each box represents the median;

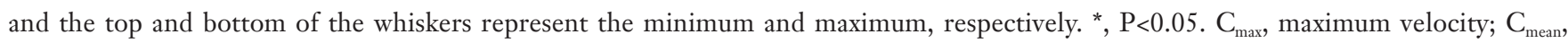
mean velocity; $\mathrm{C}_{\min }$, minimum velocity; $\mathrm{E}_{\max }$, maximum elastic modulus; $\mathrm{E}_{\text {mean }}$, mean elastic modulus; $\mathrm{E}_{\min }$, minimum elastic modulus; $\mathrm{L}$, longitudinal section; PWS, port-wine stain; T, transverse section.

\section{High-frequency US examination}

High-frequency US has high resolution, which allows the observation of the skin and its appendages, subcutaneous tissue, and deep structures. It has been successfully used to evaluate skin lesions, such as sclerosis, psoriasis, and skin tumors (16). Combined with a Doppler blood signal, high- frequency US could detect dermis hypoechogenicity and skin nodularity, measure the skin thickness, and evaluate the blood flow in PWS patients. By now, only a few studies have reported the use of high-frequency US in evaluating PWS lesions.

The present study found that the dermis hypoechogenicity, 
Table 3 Comparisons of heightened SWE values in the transverse section among the groups

\begin{tabular}{|c|c|c|c|c|c|}
\hline Heightened SWE values & Normal skin $(n=238)$ & Pink type $(n=118)$ & Purple type $(n=50)$ & Thickened type $(n=58)$ & Nodular type $(n=12)$ \\
\hline Heightened $\mathrm{E}_{\min }(\mathrm{Kpa})$ & $0.00 \pm 0.00$ & $0.43 \pm 7.14$ & $0.30 \pm 8.26$ & $0.70 \pm 5.83$ & $0.13 \pm 6.88$ \\
\hline Heightened $\mathrm{E}_{\max }(\mathrm{Kpa})$ & $0.00 \pm 0.00$ & $0.49 \pm 9.48$ & $0.76 \pm 9.94$ & $2.12 \pm 10.74^{8}$ & $5.14 \pm 5.24^{\varepsilon, *, \#}$ \\
\hline Heightened $\mathrm{C}_{\text {mean }}(\mathrm{m} / \mathrm{s})$ & $0.00 \pm 0.00$ & $0.04 \pm 0.47$ & $0.06 \pm 0.58$ & $0.11 \pm 0.49$ & $0.14 \pm 0.44$ \\
\hline Heightened $\mathrm{C}_{\max }(\mathrm{m} / \mathrm{s})$ & $0.00 \pm 0.00$ & $0.04 \pm 0.54$ & $0.06 \pm 0.63$ & $0.17 \pm 0.60^{\&}$ & $0.40 \pm 0.36^{\alpha_{, *, \#}}$ \\
\hline
\end{tabular}

\&, $\mathrm{P}<0.05$ versus the normal-skin group; ${ }^{*}, \mathrm{P}<0.05$ versus the pink-type group; ${ }^{*}, \mathrm{P}<0.05$ versus the purple-type group. SWE, shear wave elastography; $\mathrm{C}_{\max }$, maximum velocity; $\mathrm{C}_{\text {mean }}$, mean velocity; $\mathrm{C}_{\min }$, minimum velocity; $\mathrm{E}_{\max }$, maximum elastic modulus; $\mathrm{E}_{\text {mean }}$, mean elastic modulus; $\mathrm{E}_{\min }$, minimum elastic modulus.

Table 4 Comparisons of ultrasonic features and SWE values between pre-treatment and post-treatment of all groups

\begin{tabular}{|c|c|c|c|c|c|c|c|c|}
\hline $\begin{array}{l}\text { Ultrasonic features } \\
\text { and SWE values }\end{array}$ & \multicolumn{2}{|c|}{ Pink type $(n=41)$} & \multicolumn{2}{|c|}{ Purple type (n=29) } & \multicolumn{2}{|c|}{ Thickened type $(n=15)$} & \multicolumn{2}{|c|}{ Nodular type $(n=5)$} \\
\hline $\begin{array}{l}\text { Dermis } \\
\text { hypoechogenicity }\end{array}$ & $30(73.17 \%)$ & $19(46.34 \%)^{8}$ & 29 (100\%) & 29 (100\%) & $15(100 \%)$ & $15(100 \%)$ & $5(100 \%)$ & $5(100 \%)$ \\
\hline Skin nodularity & - & - & - & - & - & - & $5(100 \%)$ & $4(80 \%)$ \\
\hline $\begin{array}{l}\text { Thickened skin } \\
(\mathrm{mm})\end{array}$ & $0.37 \pm 0.27$ & $0.23 \pm 0.19^{\&}$ & $0.71 \pm 0.51$ & $0.47 \pm 0.41^{\&}$ & $0.94 \pm 0.72$ & $0.71 \pm 0.58^{\&}$ & $3.69 \pm 3.77$ & $1.60 \pm 0.92^{\&}$ \\
\hline \multicolumn{9}{|l|}{ PD signal grades } \\
\hline 0 & 36 & 36 & 20 & 20 & 4 & 6 & 1 & 1 \\
\hline 1 & 4 & 5 & 0 & 1 & 1 & 7 & 0 & 1 \\
\hline 2 & 1 & 0 & 6 & 8 & 8 & 2 & 1 & 2 \\
\hline $\begin{array}{l}\text { Heightened } \mathrm{C}_{\max } \\
(\mathrm{m} / \mathrm{s})\end{array}$ & $0.17 \pm 0.70$ & $0.16 \pm 0.45$ & $0.25 \pm 0.49$ & $0.22 \pm 0.89$ & $-0.66 \pm 2.42$ & $-0.01 \pm 0.85$ & $0.67 \pm 0.84$ & $0.27 \pm 1.81$ \\
\hline
\end{tabular}

thickness of thickened skin, and skin PD signal grades were significantly higher in all PWS-type groups compared with the normal-skin group. This indicated that high-frequency US abnormalities could be detected in most PWS lesions. Pathologically, PWS lesions were a result of vascular malformations characterized by ectatic vessels in the papillary and upper reticular dermis. Hence, corresponding grayscale ultrasonic manifestations (dermis hypoechogenicity and thickened skin) and PD ultrasonic manifestations (skin PD signals) were detected in PWS lesions. These were in line with previous imaging studies in which the PWS skin was thickened, and many enlarged vessels and increased blood flow were detected in the dermis compared with the normal contralateral skin $(6,17)$. It is worth mentioning that dermatologists categorize pink-type and purple-type lesions into flat lesions because the skin thickness observed by the naked eye in these two PWS-type lesions and normal skin showed no difference. Nevertheless, a significantly thickened skin was detected in these two flat lesions using highfrequency US compared with the normal skin.

Second, skin nodularity was detected in only nodulartype PWS skin lesions, and the hypoechoic nodule was detected in every nodular-type lesion. These coincide with the clinical manifestations that nodular-type lesions display 
deeper color and increases the risk of spontaneous bleeding. Clinically, nodules are red or dark brown, and often painless, which may be regarded as hemangioma. Histologically, nodules include nodular aggregates of vessels, exaggerated vascular ectasia, and thick-walled vessels in the dermis (18). Since high-frequency US could detect PWS nodules and hemangiomas, it could perhaps be used to differentiate nodular-type PWS lesions and hemangiomas. In our future studies, we will endeavor to investigate this.

Third, the thickened skin and skin PD signal grades in the nodular-type and thickened-type groups were significantly thicker and higher than those in the pinktype and purple-type group. As the more serious vascular malformations in these two types of lesions, thicker skin and more blood signals were detected by high-frequency US. Dermatologists occasionally categorize these two types of lesions into thickened ones. Our results were in accordance with the former US study on PWS lesions in which the thickened skin and vessel density of nodular PWSs were thicker and higher than those of flat ones (6). Moreover, these may be explained by the clinical manifestations indicating that nodular-type and thickened-type lesions could develop from the pink-type and purple-type lesions.

Fourth, although the difference of the thickened skin between purple-type and pink-type groups was not significant, the PD signal grades in purple-type groups were significantly higher than those in the pink-type group. We guess this is because purple-type lesions have more serious vascular malformations, and these results could explain why purple-type lesions display deeper color than pink-type lesions in appearance.

\section{SWE examination}

SWE allows for the assessment of skin elasticity properties and possesses several advantages, such as real-time gray-scale imaging, lack of operator dependency, and quantitative measurements (11-18). Therefore, it may help to differentiate different types of PWS skin lesions and the normal skin. A previous study confirmed that SWE had high intra- and interobserver repeatability for normal-skin elasticity (14).

On the one hand, a former study proved that SWE values of the normal skin between the transverse and longitudinal sections showed no differences (14). This meant that anisotropy was not obvious on the normal skin. Nevertheless, in this study, all SWE values ( $\mathrm{E}_{\text {mean }}$, $\mathrm{E}_{\min }, \mathrm{E}_{\text {max }}, \mathrm{C}_{\text {mean }}, \mathrm{C}_{\min }$, and $\mathrm{C}_{\max }$ ) of PWS lesions were significantly higher in the transverse section than those in the longitudinal section. Skin elasticity exhibits very large scatter, which depends on the location and skin fiber orientation $(19,20)$. Compared with the normal skin, the anatomic geometry and histopathological structure of PWS skin lesions changed, which led to skin anisotropy. Thus, differences in skin stiffness metrics $\left(\mathrm{E}_{\text {mean }}, \mathrm{E}_{\min }, \mathrm{E}_{\text {max }}, \mathrm{C}_{\text {mean }}\right.$, $\mathrm{C}_{\min }$, and $\mathrm{C}_{\max }$ ) between the transverse and longitudinal sections were derived.

On the other hand, SW velocities and Young's elastic modulus were all significantly higher in the transverse section than in the longitudinal section. Hence, the transverse sectional SWE values were chosen to compare heightened SWE values among the groups. All heightened SWE values in all PWS-type groups were higher than those in the normal-skin group. However, the differences in heightened $\mathrm{E}_{\text {mean }}, \mathrm{E}_{\text {min }}, \mathrm{C}_{\text {mean }}$, and $\mathrm{C}_{\min }$ values among the groups were not significant. However, the heightened $\mathrm{E}_{\max }$ and $\mathrm{C}_{\max }$ in the nodular-type PWS group were the highest among all the groups and significantly higher than those in the normal-skin group and pink-type and purple-type PWS groups. Meanwhile, the heightened $\mathrm{E}_{\max }$ and $\mathrm{C}_{\max }$ were significantly higher in the thickened-type PWS group than in the normal-skin group. As mentioned earlier, the nodular-type and thickened-type lesions could develop from pink-type and purple-type PWSs, and more serious vascular malformations and histopathological structural changes occurred in the dermis. Therefore, skin elasticities of these two types of PWSs were relatively higher, and the differences were statistically significant. The results also indicated that $\mathrm{E}_{\max }$ and $\mathrm{C}_{\max }$ performed relatively better in diagnosing PWS lesions, which was in agreement with previous studies $(21,22)$ in which $\mathrm{E}_{\max }$ was the bestperforming quantitative SWE feature in diagnosing breast tumors and gouty arthritis.

At last, in the evaluation of therapeutic effects, after one-time photodynamic therapy, the ratio of dermis hypoechogenicity in pink-type lesions significantly decreased, and thickened skins in all groups were significantly thinned. However, the differences of PD signal grades, heightened $\mathrm{E}_{\max }$ and $\mathrm{C}_{\max }$ in all groups between pre-treatment and post-treatment showed no significance. Our study results indicate that thickened skin is the best feature for assessing PWS patients' therapeutic effect which suggests that the thickened skin in high-frequency US is suitable to assess the therapeutic effect of PWSs and help to choose suitable treatments. However, it should be acknowledged that the sample sizes after treatment were 
relatively small, and the thus results should be subjected to further confirmation. Secondly, our study did not compare US and SWE findings with standardized visual scores. Meanwhile, only those patients who received one-time photodynamic therapy were included. These limitations should be addressed in future studies.

\section{Conclusions}

High-frequency US and SWE show feasibility and application values assessing PWS skin lesions. Their features include dermis hypoechogenicity, thicker skin, higher PD signal grades, higher $\mathrm{E}_{\max }$, and higher $\mathrm{C}_{\max }$, with thicker skin being the best feature for assessing therapeutic effect.

\section{Acknowledgments}

Funding: This study was supported by the National Natural Science Foundation of China (81971622).

\section{Footnote}

Conflicts of Interest: The authors have no conflicts of interest to declare.

Ethical Statement: The authors are accountable for all aspects of the work in ensuring that questions related to the accuracy or integrity of any part of the work are appropriately investigated and resolved. The study was approved by the West China Hospital of Sichuan University Ethics Committee. Informed consent was obtained from all volunteers.

\section{References}

1. Chen JK, Ghasri P, Aguilar G, et al. An overview of clinical and experimental treatment modalities for port wine stains. J Am Acad Dermatol 2012;67:289-304.

2. Ernemann U, Kramer U, Miller S, et al. Current concepts in the classification, diagnosis and treatment of vascular anomalies. Eur J Radiol 2010;75:2-11.

3. Brauer JA, Geronemus RG. Single-treatment resolution of vascular blebs within port wine stains using a novel 1,064$\mathrm{nm}$ neodymium-doped yttrium aluminum garnet laser. Dermatol Surg 2013;39:1113-5.

4. Flors L, Leiva-Salinas C, Maged IM, et al. MR imaging of soft-tissue vascular malformations: diagnosis, classification, and therapy follow-up. Radiographics 2011;31:1321-40.
5. Sharif SA, Taydas E, Mazhar A, et al. Noninvasive clinical assessment of port-wine stain birthmarks using current and future optical imaging technology: a review. Br J Dermatol 2012;167:1215-23.

6. Gong X, Yu W, Li J, et al. High-Frequency Ultrasound Investigation of Port-Wine Stains: Hemodynamic Features Revealed By 10- and 22-MHz Transducers. J Ultrasound Med 2019;38:641-8.

7. Chen Q, Xiao B, Merlin D. Low-frequency ultrasound may improve drug penetration in colonic mucosa. Transl Cancer Res 2017;6:S276-9.

8. Hou Y, Zhu QL, Liu H, et al. A preliminary study of acoustic radiation force impulse quantification for the assessment of skin in diffuse cutaneous systemic sclerosis. J Rheumatol 2015;42:449-55.

9. Bouhemad B, Dransart-Rayé O, Mojoli F, et al. Lung ultrasound for diagnosis and monitoring of ventilatorassociated pneumonia. Ann Transl Med 2018;6:418.

10. Wang L, Yan F, Yang Y, et al. Quantitative Assessment of Skin Stiffness in Localized Scleroderma Using Ultrasound Shear-Wave Elastography. Ultrasound Med Biol 2017;43:1339-47.

11. Yang Y, Yan F, Wang L, et al. Quantification of skin stiffness in patients with systemic sclerosis using real-time shear wave elastography: a preliminary study. Clin Exp Rheumatol 2018;36:118-25.

12. Yang Y, Qiu L, Wang L, et al. Quantitative Assessment of Skin Stiffness Using Ultrasound Shear Wave Elastography in Systemic Sclerosis. Ultrasound Med Biol 2019;45:902-12.

13. Yang Y, Wang L, Yan F, et al. Determination of Normal Skin Elasticity by Using Real-time Shear Wave Elastography. J Ultrasound Med 2018;37:2507-16.

14. Xiang X, Yan F, Yang Y, et al. Quantitative Assessment of Healthy Skin Elasticity: Reliability and Feasibility of Shear Wave Elastography. Ultrasound Med Biol 2017;43:445-52.

15. Adler DD, Carson PL, Rubin JM, et al. Doppler ultrasound color flow imaging in the study of breast cancer: preliminary findings. Ultrasound Med Biol 1990;16:553-9.

16. Barcaui EO, Carvalho AC, Lopes FP, et al. High frequency ultrasound with color Doppler in dermatology. An Bras Dermatol 2016;91:262-73.

17. Liu G, Jia W, Nelson JS, et al. In vivo, highresolution, three-dimensional imaging of port wine stain microvasculature in human skin. Lasers Surg Med 2013;45:628-32.

18. Finley JL, Noe JM, Arndt KA, et al. Port-wine stains. 
Morphologic variations and developmental lesions. Arch Dermatol 1984;120:1453-5.

19. Luo CC, Qian LX, Li GY, et al. Determining the in vivo elastic properties of dermis layer of human skin using the supersonic shear imaging technique and inverse analysis. Med Phys 2015;42:4106-15.

20. Ní Annaidh A, Bruyère K, Destrade $M$, et al.

Characterization of the anisotropic mechanical properties of excised human skin. J Mech Behav Biomed Mater 2012;5:139-48.

21. Tang Y, Yan F, Yang Y, et al. Value of Shear Wave Elastography in the Diagnosis of Gouty and Non-Gouty Arthritis. Ultrasound Med Biol 2017;43:884-92.

22. Wang ZL, Li JL, Li M, et al. Study of quantitative elastography with supersonic shear imaging in the diagnosis of breast tumours. Radiol Med 2013;118:583-90.
Cite this article as: Tang Y, Cheng S, Tang X, Guo R, Zhang L, Qiu L. Quantification of skin lesions using high-frequency ultrasound and shear wave elastography in port-wine stain patients: a clinical study. Ann Transl Med 2019;7(24):803. doi: 10.21037/atm.2019.12.57 